\title{
Imaging self-sputtering and backscattering from the substrate during pulsed laser deposition of gold
}

\author{
J. Gonzalo,* J. Siegel, A. Perea, D. Puerto, V. Resta, M. Galvan-Sosa, and C. N. Afonso \\ Laser Processing Group, Instituto de Optica, CSIC, Serrano 121, 28006 Madrid, Spain
}

(Received 21 February 2007; revised manuscript received 16 May 2007; published 27 July 2007)

\begin{abstract}
The expansion dynamics of the plasma generated during pulsed laser deposition of gold in vacuum has been investigated at the laser fluences of $2.5,6.0$, and $9.0 \mathrm{~J} \mathrm{~cm}^{-2}$. A severe distortion of the expansion is observed in the presence of a substrate that is accompanied at $9.0 \mathrm{~J} \mathrm{~cm}^{-2}$ by the appearance of a secondary plasma front expanding from the substrate surface. Langmuir probe analysis at $9.0 \mathrm{~J} \mathrm{~cm}^{-2}$ shows that the substrate surface is bombarded by a high transient flux of energetic $\mathrm{Au}^{+}$ions $\left(3.0 \times 10^{19}\right.$ ions $\left.\mathrm{cm}^{-2} \mathrm{~s}^{-1}\right)$ having very large kinetic energies $(>400 \mathrm{eV})$. Analysis of the plasma dynamics shows that these observations are consistent with self-sputtering of Au neutrals from the substrate induced by incident Au ions while a fraction of them are backscattered. Self-sputtering is found to be 2 orders of magnitude larger than backscattering. The comparison with experimental data allows concluding that the apparent recoil of the plasma front is caused by collision with self-sputtered neutrals, while the secondary emission is originated by backscattered ions.
\end{abstract}

DOI: 10.1103/PhysRevB.76.035435

PACS number(s): 68.55.Ac, 81.15.Fg, 52.70.Kz, 52.70.Ds

\section{INTRODUCTION}

Pulsed laser deposition (PLD) has become a well established technique for the production of a broad variety of materials, particularly complex oxides. ${ }^{1,2}$ However, much less attention has been paid to the production of metals despite early attempts to synthesize them in the 1970 s. ${ }^{3}$ More recently, the interest in complex thin film metal nanostructures such as metal-dielectric or metal multilayers, ${ }^{4,5}$ as well as metal nanoparticles, ${ }^{6-8}$ has triggered new efforts to use PLD for metal deposition. These metal structures are characterized by the localization and enhancement of the electric and magnetic fields, leading to effects such as giant magnetoresistance, ${ }^{9}$ enhanced nonlinear optical properties, ${ }^{8}$ or enhanced Raman response, ${ }^{10}$ among others. Layer thickness, bulk and interface structure in the case of the multilayers, or size, shape, and arrangement of nanoparticles determine these properties. Thus, practical applications require excellent control of the structure and morphology of the nanostructures.

PLD is characterized by a high instantaneous flux of species reaching the substrate, a fraction of them having high kinetic energies. ${ }^{11-14}$ Both characteristics are expected to have a strong influence on the features of the metal structures produced. ${ }^{6,15}$ In addition, the presence of highly energetic species may induce undesired processes such as selfsputtering, also termed resputtering, of a fraction of the material that is being deposited ${ }^{14,16-19}$ or an increase of surface roughness, ${ }^{4}$ among others. Finally, both self-sputtering and backscattering of a fraction of the incident species may have a significant effect on the morphology of the nanoparticles due to their nanometric dimensions. While self-sputtering is considered to be a common process when PLD is performed in vacuum, backscattering has seldom been observed or discussed in the literature, ${ }^{20}$ and little effort has been devoted to determine its influence on the properties of the deposited films. Most of these studies can be grouped into two types. Either the stoichiometry and amount of material deposited in different substrate configurations are analyzed ${ }^{16,17}$ to quan- tify the extent of resputtering ${ }^{11}$ or the expansion dynamics of laser generated plasmas is studied, and self-sputtering yields are calculated from the measured velocity distributions. ${ }^{12,14}$ However, no systematic studies have been performed in the presence of a substrate. Van de Riet et al. ${ }^{16}$ combined both approaches to correlate the compositional changes observed in FeSiGaRu films with its bombardment by energetic ions present in the plasma. Kummuduni and co-workers identified the presence of YO molecules and $\mathrm{Ba}$ atoms moving backward from the substrate during the growth of $\mathrm{YBaCuO}$ films by PLD and measured the velocity of Ba atoms. ${ }^{20,21}$ Finally, Geohegan observed the emission of species near the substrate that moved toward the target following the ablation of an $\mathrm{YBaCuO}$ target. $^{22}$ Except for Van de Riet et al., none of them correlated their experimental observations with selfsputtering or backscattering.

The aim of this work is to determine the importance of self-sputtering and backscattering on pulsed laser deposition of metals. Analysis of Au plasma expansion dynamics in the presence of a substrate shows a severe distortion and the appearance of a secondary emission near the substrate. $\mathrm{Nu}-$ merical evaluation of self-sputtering and backscattering at the substrate surface shows that these phenomena are the signature of resputtered Au neutrals from the substrate and of $\mathrm{Au}$ ions backscattered by the substrate.

\section{EXPERIMENT}

Gold films have been produced by PLD in vacuum $\left(10^{-4} \mathrm{~Pa}\right)$ using an $\mathrm{ArF}$ excimer laser $(\lambda=193 \mathrm{~nm}, \tau=20 \mathrm{~ns}$ full width at half maximum). The laser beam was focused at the surface of a rotating $\mathrm{Au}(99.99 \%)$ target at an angle of incidence of $45^{\circ}$. The films were grown on glass substrates placed $39 \mathrm{~mm}$ from the target surface held at room temperature. The laser fluences were well above the plasma formation threshold, namely, 2.5, 6.0, and $9.0 \mathrm{~J} \mathrm{~cm}^{-2}$. The laser spot size at the target surface was kept constant and close to $1 \mathrm{~mm}^{2}{ }^{6}$ 
The plasma generated by the laser pulse was imaged on a time-gated ICCD camera (spectral range from 180 to $870 \mathrm{~nm}, 512 \times 512$ pixels, an effective pixel size of $24 \mu \mathrm{m}$, an analog to digital resolution of $16 \mathrm{bits}$, and a minimum gate width of $2 \mathrm{~ns}$ ) at an angle of $90^{\circ}$ with respect to the surface normal using a camera lens (focal length, $50 \mathrm{~mm} ; f$ number, $f / 1.8)$. The time gate of the ICCD was delayed with respect to the laser pulse in order to record the complete evolution of the plasma expansion. The synchronization, delay, gate width, and image acquisition were controlled via software. ${ }^{23}$ Finally, a bandpass filter (central wavelength $=490 \mathrm{~nm}$, bandwidth $=38 \mathrm{~nm}$ ) was installed in front of the ICCD camera to ensure that the plasma emission recorded was mainly due to the two dominant emission lines of excited $\mathrm{Au}$ neutrals $\left(\mathrm{Au}^{*}\right)$ : $479.3 \mathrm{~nm} \quad\left(5 d^{10} 6 d^{2} D_{5 / 2}-5 d^{10} 6 p^{2} P_{3 / 2}^{0}\right) \quad$ and $481.2 \mathrm{~nm}$ $\left(5 d^{10} 6 d^{2} D_{3 / 2}-5 d^{10} 6 p^{2} P_{3 / 2}^{0}\right) .{ }^{24}$ The contribution of the $\mathrm{Au}^{+*}$ emission line at $476.0 \mathrm{~nm}$ that corresponds to the transition, $5 d^{8} 6 s^{2}{ }^{2} 8_{3}-5 d^{9} 6 p 4_{2}^{0},{ }^{24}$ is expected to be marginal since its tabulated intensity is only $0.6 \%$ and $3 \%$ of the 479.3 and $481.2 \mathrm{~nm} \mathrm{Au}^{*}$ lines. Before any time-gated image was acquired, a thin (approximately $50 \mathrm{~nm}$ ) gold film was deposited at the substrate to ensure that the imaging experiments were performed under similar surface conditions.

The density and velocity distributions of $\mathrm{Au}^{+}$ions in the plasma were determined using a Langmuir probe having a sensitive area of $\approx 2.0 \pm 0.1 \mathrm{~mm}^{2}$ placed at the substrate position. The Langmuir probe was biased at a voltage of $-10 \mathrm{~V}$ to ensure that only ions and not electrons were collected, while its rear side was electrically insulated using a Teflon sheet. Real time current transients were collected by a Koopman circuit connected to a digital oscilloscope. Two laser pulses separated by $1 \mathrm{~s}$ were used: the first one to clean the target surface and the second one for recording the current transient. Finally, the density of $\mathrm{Au}^{+}$ions was determined by integrating the total charge collected per laser pulse and normalizing it by the probe area.

\section{RESULTS}

Figure 1 shows a series of time-gated images of expanding $\mathrm{Au}^{*}$ species generated at $9.0 \mathrm{~J} \mathrm{~cm}^{-2}$ at increasing time delays $t$ with respect to the laser pulse. Each image was obtained by accumulating over ten laser pulses with a constant gate width of $\Delta t=100 \mathrm{~ns}$. The plasma emission intensity is represented in a false color scale and the intensity of each image has been normalized to the brightest pixel. The positions of the target, substrate, and substrate holder have been included in the images. Shortly after the laser pulse $(t=1.5 \mu \mathrm{s})$, the plasma expands along the target surface normal (primary emission). As $t$ increases, the plasma keeps expanding and a secondary emission appears close to the substrate, even before the front of $\mathrm{Au}^{*}$ distribution reaches its surface $(t=2.5 \mu \mathrm{s})$. At longer delays, the primary emission becomes distorted, and its front seems to stop $(t=4.0 \mu \mathrm{s})$ and to be even driven backward toward the target surface $(t=5.0 \mu \mathrm{s}$ and $t=6.5 \mu \mathrm{s})$. We have confirmed that the secondary emission and the perturbation of the primary
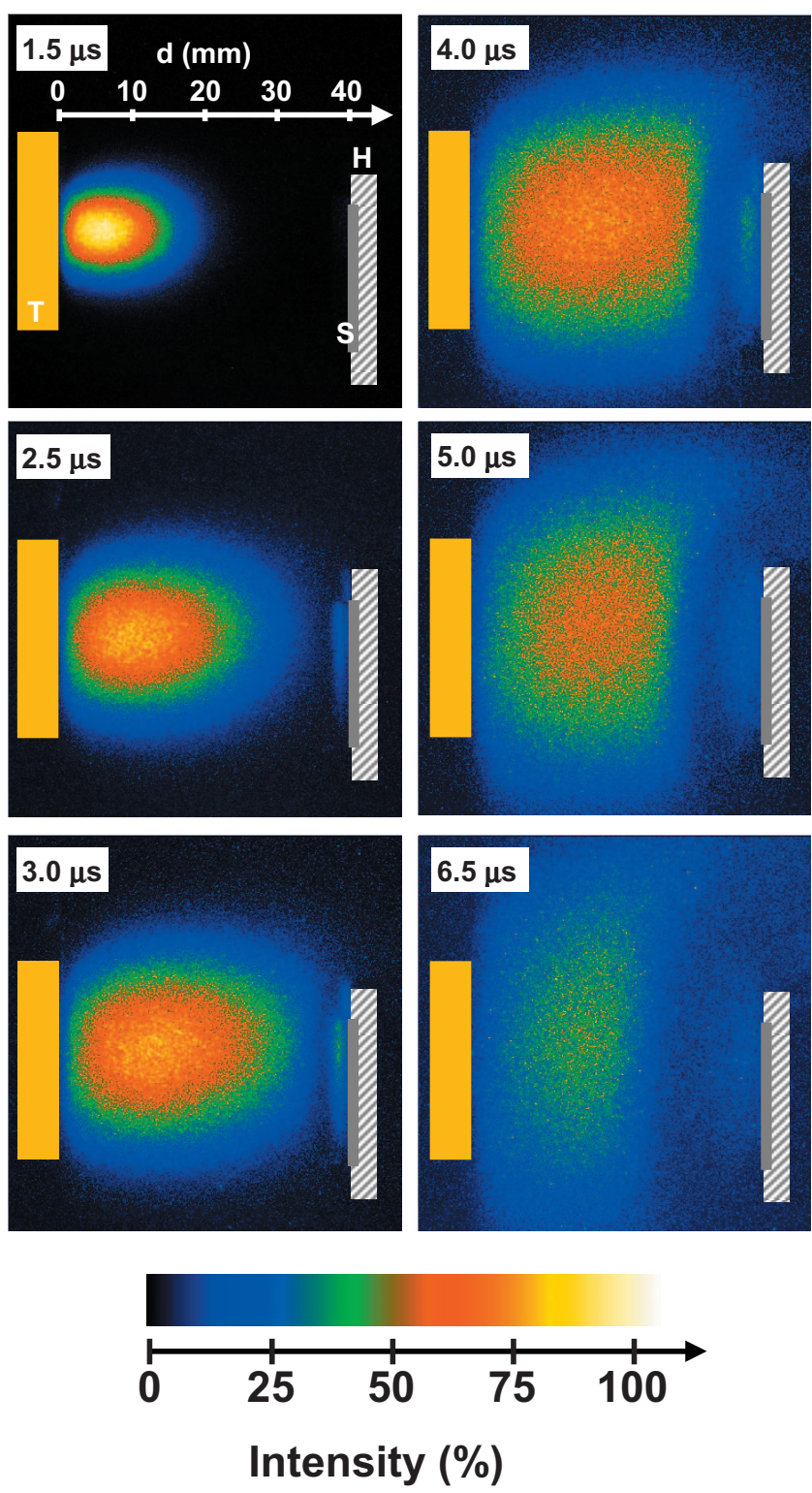

FIG. 1. (Color) Two dimensional time-gated images of the $\mathrm{Au}$ plasma generated at $9.0 \mathrm{~J} \mathrm{~cm}^{-2}$ that corresponds to the emission of excited $\mathrm{Au}$ neutrals at 479.3 and $481.2 \mathrm{~nm}$. The emission intensity is represented in false colors and the data have been normalized by the maximum intensity for each time delay. The time delays with respect to the laser pulse are indicated in each image. The relative position of (T) target, (S) substrate, and (H) substrate holder are schematically shown in each image. The origin of the plasma expansion $(z=0)$ is the target surface.

emission are not observed in the absence of substrate and substrate holder.

The transient plasma emission intensity distributions along the expansion axis have been extracted from each time-gated image by averaging over 10 pixel rows to improve the signal to noise ratio. They are shown in Fig. 2 for $9.0 \mathrm{~J} \mathrm{~cm}^{-2}$. The data are normalized to the maximum intensity for each time delay. At $t=1.5 \mu \mathrm{s}$, we observe a single peak related to the primary emission that extends up to $20 \mathrm{~mm}$ from the target surface, the maximum intensity being 


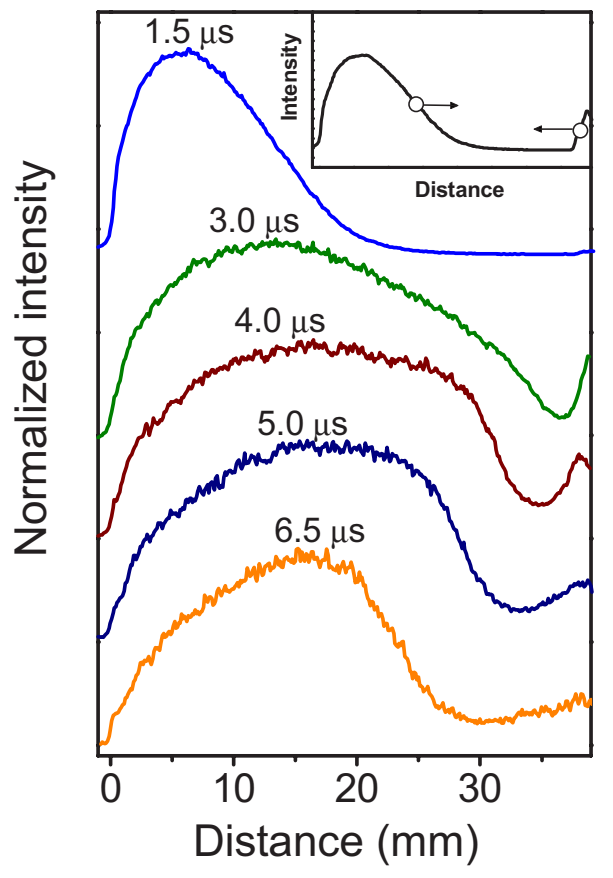

FIG. 2. (Color online) Temporal evolution of the spatial distribution of $\mathrm{Au}^{*}$ emitting at 479.3 and $481.2 \mathrm{~nm}$ generated at $9.0 \mathrm{~J} \mathrm{~cm}^{-2}$. The time delays with respect to the laser pulse are indicated in the figure. The data have been normalized by the maximum intensity for each time delay. The inset schematically shows the spatial positions of the intensity spatial distributions, where the primary and secondary emission reach half of their maximum value. The temporal evolution of these positions is considered to be representative of the movements of the primary and secondary fronts.

observed at $6 \mathrm{~mm}$. At longer delays $2.5 \leqslant t \leqslant 5.0 \mu \mathrm{s}$, both primary and secondary emissions are clearly observed. The primary emission broadens as $t$ increases, up to $t=3.0 \mu \mathrm{s}$, and it narrows for longer delays. The secondary emission also reaches its maximum at $t=3.0 \mu \mathrm{s}$, while it broadens and decreases its relative intensity for longer delays. Moreover, the analysis of the secondary emission reveals that it moves toward the target. The primary emission presents similar features in the case of plasmas generated at 6.0 and $2.5 \mathrm{~J} \mathrm{~cm}^{-2}$, while the secondary emission is found to be very weak and not appreciable, respectively.

In order to characterize in detail the Au plasma expansion dynamics, we have analyzed the temporal evolution of the primary and secondary emission fronts, $D$, defined as the position where the primary and secondary emission reach half of their maximum values. This is schematically indicated in the inset of Fig. 2 and will be referred to hereafter as primary and secondary fronts. Figure 3 shows their dependence on the time delay $t$ for 2.5 and $9.0 \mathrm{~J} \mathrm{~cm}^{-2}$. In the case of the primary front, $D(t)$ shows a linear increase up to a delay that depends on fluence: $t_{\max }=3.5 \mu \mathrm{s}$ for $9.0 \mathrm{~J} \mathrm{~cm}^{-2}$ and $t_{\max }=4.5 \mu \mathrm{s}$ for $2.5 \mathrm{~J} \mathrm{~cm}^{-2}$. After this maximum expansion, $D_{\max }$, the primary front moves backward (i.e., recoils), as the negative slope in Fig. 3 shows. The expansion $v_{p \text {,exp }}$ and recoil $v_{p \text {,recoil }}$ velocities of the primary front can be estimated from the slopes of $D(t)$. They increase with laser fluence, whereas $t_{\max }$ decreases. These results are included in Fig. 3 and in Table I.



FIG. 3. (Color online) Space-time evolution of the primary and secondary emission fronts. Results obtained at (open symbols) $2.6 \mathrm{~J} \mathrm{~cm}^{-2}$ and (full symbols) $9.0 \mathrm{~J} \mathrm{~cm}^{-2}$ are included. ( $\square, \boldsymbol{\square}$ ) Primary fronts expanding from the target, $(\triangle, \mathbf{\Delta})$ primary fronts moving toward the target, and $(\bullet)$ secondary plasma front. No secondary emission is observed at $2.6 \mathrm{~J} \mathrm{~cm}^{-2}$. The top horizontal axis corresponds to the substrate surface position $(d=39 \mathrm{~mm})$. Dashed lines are linear fits to the experimental data. Dotted lines correspond to the linear extrapolation toward the substrate surface of the primary front moving toward the target and the secondary plasma front. The full line represents the space-time evolution of Au species expanding from the target with a velocity of $27.3 \mathrm{~km} \mathrm{~s}^{-1}$. The numbers in the figure correspond to the absolute velocities determined from the slopes of the linear fits of the experimental data.

The secondary emission front, observed at $9.0 \mathrm{~J} \mathrm{~cm}^{-2}$, shows also a linear dependence, with the front moving toward the target. Its recoil velocity $v_{s, \text { recoil }}$ is $-0.8 \mathrm{~km} \mathrm{~s}^{-1}$, which is almost four times smaller than the corresponding $v_{p, \text { recoil }}$. As shown in Fig. 3, the onset for observation of the secondary plasma emission occurs $2 \mu$ s after the laser pulse, which is much earlier than the time when the primary emission front changes its apparent sense of movement $\left(t_{\max }=3.5 \mu \mathrm{s}\right)$.

The density of $\mathrm{Au}^{+}$ions per pulse is also included in Table I. Values of the order of $10^{14}$ ions $\mathrm{cm}^{-2}$ are measured and they increase by a factor of 5 when the fluence increases from 2.5 to $9.0 \mathrm{~J} \mathrm{~cm}^{-2}$. The velocity distributions of $\mathrm{Au}^{+}$, $N(v)$, have been calculated from the current transients, $F(t)$, using the relationship $v=d / t$, where $d$ is the position of the probe with respect to the target surface $(d=39 \mathrm{~mm})$ and $t$ the time delay with respect to the laser pulse. The Jacobian $J(v)=t^{2} / d$ has been considered for the transformation ${ }^{16}$ $N(v)=F(t)|J(v)|$. The normalized velocity distributions of $\mathrm{Au}^{+}$determined for 2.5 and $9.0 \mathrm{~J} \mathrm{~cm}^{-2}$ are shown in Fig. 4. Whereas the most probable velocity of ions (i.e., peak of the distribution) shows only a small increase with fluence, the fraction of ions having large velocities $\left(v>20 \mathrm{~km} \mathrm{~s}^{-1}\right)$ increases considerably. The characteristic front velocities for which $\mathrm{Au}^{+}$distributions reach their half-maximum value $\left(v_{\text {ion }}\right)$ are included in Table I. The corresponding normalized velocity distributions of $\mathrm{Au}^{*}$ are also included in Fig. 4. They have been determined from the transient plasma emission intensity recorded at $1.0 \mu \mathrm{s}$. This delay was chosen because no distortion of the plasma is observed. Excited neutrals are confined to smaller velocity values than ions for both flu- 
TABLE I. Characteristic kinetic data relative to Au plasma expansion extracted from optical emission spectroscopy and Langmuir probe measurements. $\left(t_{\max }\right)$ Time delay when the primary emission front of Au neutrals reaches its maximum expansion (i.e., when the primary front starts moving backward), $\left(v_{p, \exp }\right)$ expansion and $\left(v_{p, \text { recoil }}\right)$ recoil velocities of the primary front, $\left(v_{s, \text { recoil }}\right)$ velocity of the secondary plasma front, $\left(v_{i o n}\right)$ characteristic front velocity of Au ions, and integrated ion density, The sign minus, "-", indicates that the front moves toward the target surface.

\begin{tabular}{lcccccc}
\hline \hline $\begin{array}{l}\text { Fluence } \\
\left(\mathrm{J} \mathrm{cm}^{-2}\right)\end{array}$ & $\begin{array}{c}t_{\text {max }} \\
(\mu \mathrm{s})\end{array}$ & $\begin{array}{c}v_{\text {pexp }} \\
\left(\mathrm{km} \mathrm{s}^{-1}\right)\end{array}$ & $\begin{array}{c}v_{\text {p,recoil }} \\
\left(\mathrm{km} \mathrm{s}^{-1}\right)\end{array}$ & $\begin{array}{c}v_{s, \text { recoil }} \\
\left(\mathrm{km} \mathrm{s}^{-1}\right)\end{array}$ & $\begin{array}{c}v_{\text {ion }} \\
\left(\mathrm{km} \mathrm{s}^{-1}\right)\end{array}$ & $\begin{array}{c}\mathrm{Au}^{+} \text {density } \\
\left(\text { ions cm }^{-2}\right)\end{array}$ \\
\hline 2.5 & 4.5 & 7.9 & -1.8 & - & 15.6 & $0.3 \times 10^{14}$ \\
6.0 & 3.6 & 9.3 & -2.7 & - & 17.4 & $0.9 \times 10^{14}$ \\
9.0 & 3.5 & 9.9 & -3.1 & -0.8 & 18.1 & $1.4 \times 10^{14}$ \\
\hline \hline
\end{tabular}

ences. Finally, it is worth noting that the fraction of neutrals having velocities $>20 \mathrm{~km} \mathrm{~s}^{-1}$ is negligible in all cases.

\section{DISCUSSION}

The results presented demonstrate that the expansion of the plasma is severely distorted by the presence of the substrate. The main effects are the apparent recoil movement of the primary front after a delay $t_{\max }$ that depends on the laser fluence and the appearance of the secondary plasma emission close to the substrate surface at the highest fluence considered (Fig. 3). Since none of these effects are observed in the absence of the substrate, they must be related to the interaction of the species present in the plasma and the substrate itself. In particular, the early appearance of the secondary emission (Fig. 3) clearly indicates that certain $\mathrm{Au}$ species reach the substrate before the arrival of the primary $\mathrm{Au}^{*}$ emission front.

In order to identify these species, we have determined the time delay at which the apparent recoil of the primary and secondary fronts starts by linearly extrapolating the data in Fig. 3 toward the substrate. The fact that both fronts intersect in space and time near the substrate $\left(t_{0, \text { recoil }}=1.84 \mu \mathrm{s}\right.$, $D_{0, \text { recoil }}=37.8 \mathrm{~mm}$ for a fluence of $9.0 \mathrm{~J} \mathrm{~cm}^{-2}$ ) suggests their common origin. Assuming a constant expansion velocity, the velocity of the responsible species is $v_{o}=27.3 \mathrm{~km} \mathrm{~s}^{-1}$. This value is consistent with $\mathrm{Au}^{+}$ions since, from the velocity distributions shown in Fig. 4, approximately $17 \%$ of the ions

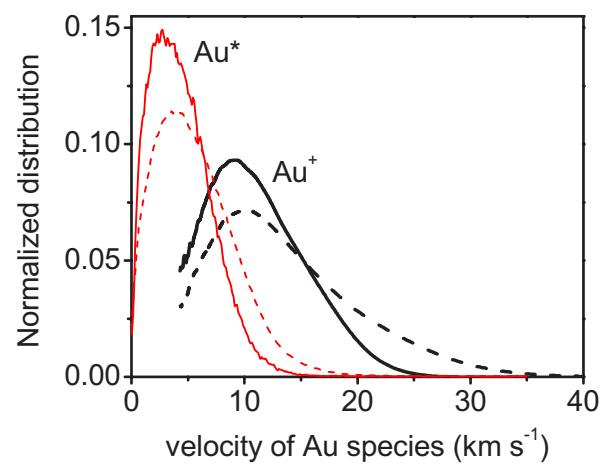

FIG. 4. (Color online) Normalized velocity distributions determined for $\mathrm{Au}^{*}$ neutrals and $\mathrm{Au}^{+}$ions at (solid line) $2.6 \mathrm{~J} \mathrm{~cm}^{-2}$ and (dashed line) $9.0 \mathrm{~J} \mathrm{~cm}^{-2}$. have velocities $>20 \mathrm{~km} \mathrm{~s}^{-1}$ (i.e., kinetic energies larger than $400 \mathrm{eV}$ ), whereas the corresponding fraction of neutrals is below $0.5 \%$. This means that after each laser pulse at $9.0 \mathrm{~J} \mathrm{~cm}^{-2}$, a large density of ions $\left(\approx 2.4 \times 10^{13}\right.$ ions $\left.\mathrm{cm}^{-2}\right)$ having kinetic energies $>400 \mathrm{eV}$ will bombard the substrate in a time of $\approx 0.8 \mu \mathrm{s}$, which leads to an approximate transient incident flux of $3.0 \times 10^{19}$ ions $\mathrm{cm}^{-2} \mathrm{~s}^{-1}$, a value much higher than those typically involved in sputtering processes. This result and the high ionization degree found in plasmas generated by UV laser ablation of noble metals ${ }^{16,17}$ suggest that $\mathrm{Au}^{+}$ions are responsible for the distortion of the plasma expansion by generating species moving opposite to the plasma expansion through self-sputtering and backscattering.

Self-sputtering occurs when the kinetic energy of the species arriving to the substrate exceeds a threshold value that for most metals is close to $25 \mathrm{eV} .{ }^{17}$ From the results presented in Fig. 4, we have estimated that $>95 \%$ of the ions satisfy this condition at the lowest fluence $\left(2.5 \mathrm{~J} \mathrm{~cm}^{-2}\right)$. Thus, we should expect significant self-sputtering of Au from the growing film at the three fluences. In addition, partial backscattering of the incident ions can occur. The extent of both processes and the nature of the species involved have been quantified using the procedure described in the Appendix, which is based in the SRIM2003 software. $^{25}$

The density distributions of self-sputtered (SS) neutrals and backscattered (BS) ions as a function of the velocity of incident $\mathrm{Au}^{+}$ions have been evaluated using expression (A2) of the Appendix, where $Q \approx 1.4 \times 10^{14}$ ions $\mathrm{cm}^{-2}$ (Table I), $N(v)$ is the normalized velocity distribution of incident $\mathrm{Au}^{+}$ measured for $9.0 \mathrm{~J} \mathrm{~cm}^{-2}$ (Fig. 4), and $Y_{S S}(v)$ and $Y_{B S}(v)$ are the average yields calculated using the procedure described in the Appendix. The results obtained are presented in Fig. 5. The half-maxima of the calculated density distributions are predicted to occur at comparable velocities of incident ions: $\approx 27.4 \pm 0.6 \mathrm{~km} \mathrm{~s}^{-1}$. This value is in very good agreement with the characteristic velocity extrapolated from Fig. 3 for the species responsible for the recoil of the primary and secondary fronts $\left(v_{0}=27.3 \mathrm{~km} \mathrm{~s}^{-1}\right)$ and thus further confirms that ions are responsible for both self-sputtering and backscattering. Using expression (A4) of the Appendix, we have evaluated the velocity distributions of SS neutrals and BS ions to determine the process responsible for the recoil of the primary emission front and the secondary emission front. The results obtained, which are presented in Fig. 6, show that SS neutrals have larger characteristic velocities $\left(v_{S S}\right.$ 


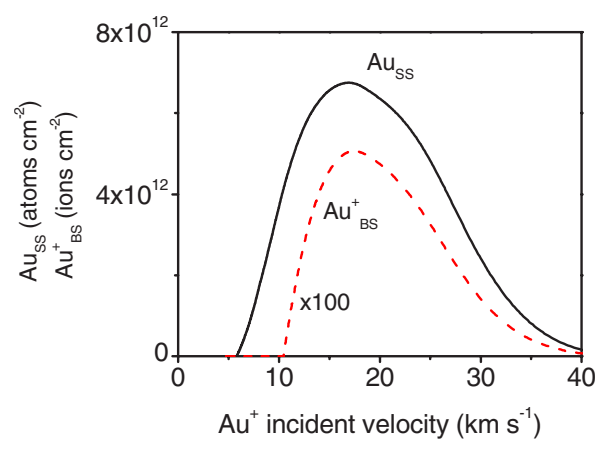

FIG. 5. (Color online) Calculated density distributions of (solid line) self-sputtered atoms, $\mathrm{Au}_{\mathrm{SS}}$, and (dashed line) backscattered ions, $\mathrm{Au}_{\mathrm{BS}}^{+}$, as a function of the velocity of $\mathrm{Au}^{+}$ions arriving to the substrate for a laser fluence of $9.0 \mathrm{~J} \mathrm{~cm}^{-2}$. Data corresponding to backscattered ions have been multiplied by a factor of 100 to ease the comparison.

$\left.=-2.6 \pm 0.1 \mathrm{~km} \mathrm{~s}^{-1}\right)$ than BS ions $\left(v_{B S}=-1.1 \pm 0.1 \mathrm{~km} \mathrm{~s}^{-1}\right)$. Taking into account the approximations considered in the modeling (see Appendix), these values are in good agreement with the experimental values determined from Fig. 3 for the recoil movement of the primary and the secondary fronts $\left(v_{p, \text { recoil }}=-3.1 \mathrm{~km} \mathrm{~s}^{-1}\right.$ and $v_{s, \text { recoil }}=-0.8 \mathrm{~km} \mathrm{~s}^{-1}$, respectively). It is then concluded that SS neutrals are responsible for the apparent recoil of the primary front, while BS ions give rise to the secondary emission.

The total density of SS neutrals and BS ions per pulse can be determined either from Fig. 5 and 6 . The density of SS neutrals $\left(\approx 1.2 \times 10^{14}\right.$ at. $\left.\mathrm{cm}^{-2}\right)$ is approximately 2 orders of magnitude larger than that of BS ions $\left(\approx 0.7 \times 10^{12}\right.$ ions $\left.\mathrm{cm}^{-2}\right)$. This ratio is maintained at low fluence $\left(2.5 \mathrm{~J} \mathrm{~cm}^{-1}\right)$ for which we have obtained values of $\approx 0.2 \times 10^{14}$ at. $\mathrm{cm}^{-2}$ and $\approx 0.1 \times 10^{12}$ ions $\mathrm{cm}^{-2}$ for SS neutrals and BS ions, respectively. While the ratio remains approximately constant, the absolute values decrease significantly when decreasing the ablation fluence, which explains why the secondary emission vanishes at low fluence.

The above considerations imply that collisions among SS $\mathrm{Au}$ atoms and excited $\mathrm{Au}^{*}$ atoms present at the front of the expanding plasma must occur before $\mathrm{Au}^{*}$ atoms reach the

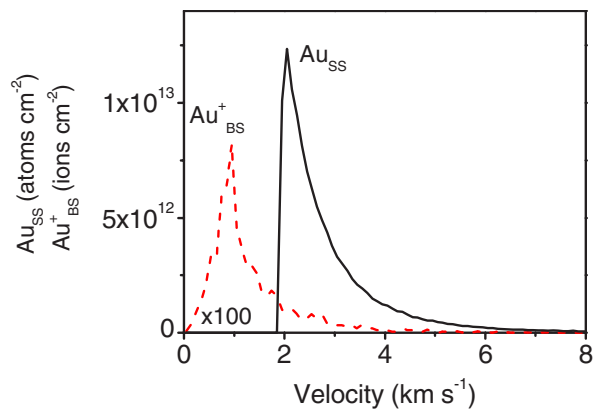

FIG. 6. (Color online) Calculated velocity distributions of (solid line) self-sputtered atoms, $\mathrm{Au}_{\mathrm{SS}}$, and (dashed line) backscattered ions, $\mathrm{Au}^{+}{ }_{\mathrm{BS}}$, for a fluence of $9.0 \mathrm{~J} \mathrm{~cm}^{-2}$. Data corresponding to backscattered ions have been multiplied by a factor of 100 to ease the comparison. substrate, and this must lead to their nonradiative deexcitation. ${ }^{26}$ Such collision-induced deexcitation processes can take place in different ways, for instance, through dimer formation as observed in laser generated sodium plasmas. ${ }^{27}$ In addition, $\mathrm{BS}$ ions could recombine into excited $\mathrm{Au}^{*}$ atoms ${ }^{26}$ in the vicinity of the substrate, thus giving rise to the secondary emission. This scenario would involve two steps, recombination and excitation. BS ions interact not only with the fraction of ions still reaching the substrate but also with the electrons. The latter could cause recombination of an ion into a neutral via electron capture as well as electron-induced excitation of the so-produced neutral. ${ }^{26}$ In addition, the possible contribution of $\mathrm{Au}^{+*}$ to the secondary emission through direct emission at $476.0 \mathrm{~nm}$ cannot completely be ruled out, since this weak emission line is not fully suppressed by the bandpass filter used. Excitation of backscattered $\mathrm{Au}^{+}$ions might occur immediately after the scattering event near the substrate through collisions with the high density of electrons arriving from the opposite direction.

\section{CONCLUSIONS}

The plasma expansion during PLD of gold shows a severe distortion evidenced by the apparent backward movement of the front of the laser generated plasma and the appearance of a secondary emission originated at the substrate. Analysis of the plasma dynamics suggests that both processes have a common origin. We provide experimental evidence supported by numerical calculations that energetic $\mathrm{Au}^{+}$ions present at the plasma front having expansion velocities larger than $20 \mathrm{~km} \mathrm{~s}^{-1}$ are responsible for both effects through selfsputtering of $\mathrm{Au}$ neutrals from the growing film and backscattering of a fraction of incident $\mathrm{Au}^{+}$ions. Self-sputtering is 2 orders of magnitude more intense than backscattering for the fluences considered $\left(2.5,6.0\right.$, and $\left.9.0 \mathrm{~J} \mathrm{~cm}^{-2}\right)$. The comparison of the calculated velocity distributions of selfsputtered neutrals and backscattered ions and the measured velocities for the recoil of the primary emission front and for the secondary emission front allows us to conclude that selfsputtered Au neutrals are responsible for the first effect, while the secondary emission is originated by backscattered $\mathrm{Au}^{+}$ions.

\section{ACKNOWLEDGMENTS}

This work was partially supported by MAT2005-06508C02-01, MEC (Spain). M.G.-S. acknowledges support from the CSIC and the European Social Fund through an I3P technical contract.

\section{APPENDIX}

Self-sputtering and backscattering induced by $\mathrm{Au}^{+}$ions during PLD of gold have been evaluated using the SRIM2003 software. ${ }^{25}$ We have considered the parameters of bulk $\mathrm{Au}$ (density $=19.11 \mathrm{~g} \mathrm{~cm}^{-3}$, lattice binding energy $=3.0 \mathrm{eV}$, surface binding energy $=3.8 \mathrm{eV}$, and displacement energy $=25 \mathrm{eV})$ (Refs. 17 and 25) to characterize the Au films and monoenergetic (single velocity) ions with kinetic energies in the range of $25-2000 \mathrm{eV}$ (i.e., velocities in the range of 




FIG. 7. (Color online) Geometry used for the self-sputtering and backscattering simulations. (Dotted arrow) Trajectory of incident $\mathrm{Au}^{+}$beam, (continuous arrow) trajectory of self-sputtered and/or backscattered species, $(\theta)$ its angle with respect to the target surface normal ( $z$ axis), and (dashed arrow) velocity component of selfsputtered and/or backscattered species along the $z$ axis, $v_{z}^{\prime}$.

$2.4-45.0 \mathrm{~km} \mathrm{~s}^{-1}$ ) as incident beams. The geometry used in the simulation is illustrated in Fig. 7. The impact point at the substrate surface is the origin of coordinates. Incident $\mathrm{Au}^{+}$ ions are considered to be normal to the surface substrate and to have the same velocity $v$ for each simulation step. This approximation is supported by the fact that strongly forward peaked angular distributions have been measured for ions generated by UV laser ablation of metals. ${ }^{13}$ This approximation eases enormously the calculation, and yet we estimate it to be representative of $\approx 50 \%$ of the ions present in the plasma that reach the substrate with an angle of incidence $\leqslant 5^{\circ}$.

SS and BS species will have different velocities $v^{\prime}$ and trajectories. SRIM simulations provide the velocity modulus and the trajectory defined by their angles with respect to the three axes $(x, y, z)$ for each species. However, since our analysis of the plasma expansion is performed along the normal to the substrate surface ( $z$ axis), we have only extracted from the simulations the velocity component along the $z$ axis (Fig. 7): $v_{z}^{\prime}=v^{\prime} \cos (\theta)$, where $\theta$ is the angle with respect to the $z$ axis.

From these data, it is possible to determine the elemental yields of SS atoms $y_{S S}\left(v, v_{z}^{\prime}\right)$ and BS ions $y_{B S}\left(v, v_{z}^{\prime}\right)$, i.e., species having normal recoil velocities $v_{z}^{\prime}$, which have been generated by incident ions having a velocity $v$. The average self-sputtering $Y_{S S}(v)$ (SS atoms/incident ion) and backscattering $Y_{B S}(v)$ (BS ions/incident ion) yields can be then calculated by integrating over $v_{z}^{\prime}$ as

$$
Y_{i}(v)=\int_{v_{z}^{\prime}} y_{i}\left(v, v_{z}^{\prime}\right) d v_{z}^{\prime},
$$

where the subindex $i$ refers to SS or BS species. $Y_{S S}(v)$ and $Y_{B S}(v)$ are presented in Fig. 8 as a function of the velocity of incident $\mathrm{Au}^{+}$ions, $v$. It is seen that $Y_{S S}(v)$ increases almost linearly in the considered velocity range and it becomes larger than 1 for $v>15.6 \mathrm{~km} \mathrm{~s}^{-1}$, while $Y_{B S}(v)$ is 2 orders of magnitude smaller and tends to saturate to a value close to 0.02 (i.e., 2 ions backscattered per 100 incident ions) for

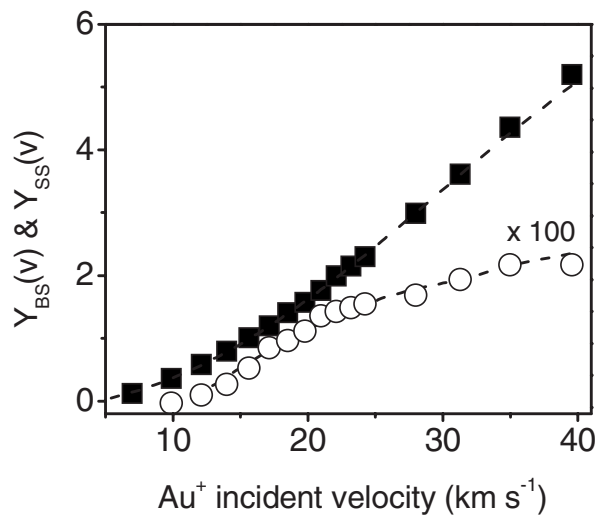

FIG. 8. Simulated (ם) self-sputtering yield of neutrals, $Y_{S S}(v)$, and $(\bigcirc)$ backscattering yield of ions, $Y_{B S}(v)$. $Y_{B S}(v)$ has been multiplied by a factor of 100 to ease the comparison. The data are presented as a function of the velocity of the $\mathrm{Au}^{+}$ions arriving to the substrate. The dashed lines are best fits to the calculated data.

$v>22.1 \mathrm{~km} \mathrm{~s}^{-1}$. The velocity thresholds to observe significant self-sputtering or backscattering are 5.4 and $10.0 \mathrm{~km} \mathrm{~s}^{-1}$, respectively. The density distributions of SS neutrals $P_{S S}(v)$ and BS ions $P_{B S}(v)$ as a function of the velocity of incident $\mathrm{Au}^{+}$ions, $v$, can be evaluated as

$$
P_{i}(v)=Q N(v) Y_{i}(v)
$$

where the subindex $i$ has the same meaning as before, $Q$ is the integrated ion density, $N(v)$ is the normalized velocity distribution of incident $\mathrm{Au}^{+}$ions, and $Y_{i}(v)$ the relevant yield. This equation allows determining the effect of the velocity of incident ions on the relative importance of SS and BS species. In addition, it is possible to determine the velocity distributions of the SS and BS species from SRIM simulations. For that purpose, we must define the elemental velocity distributions of SS atoms and BS ions, $s_{i}\left(v, v_{z}^{\prime}\right)$, i.e., the fraction of species having a normal recoil velocity $v_{z}^{\prime}$, generated by incident ions with velocity $v$, as

$$
s_{i}\left(v, v_{z}^{\prime}\right)=Q N(v) y_{i}\left(v, v_{z}^{\prime}\right),
$$

where the subindex $i, Q, N(v)$, and $y_{i}\left(v, v_{z}^{\prime}\right)$ have the same meaning as before. This is illustrated in Fig. 9 that shows the

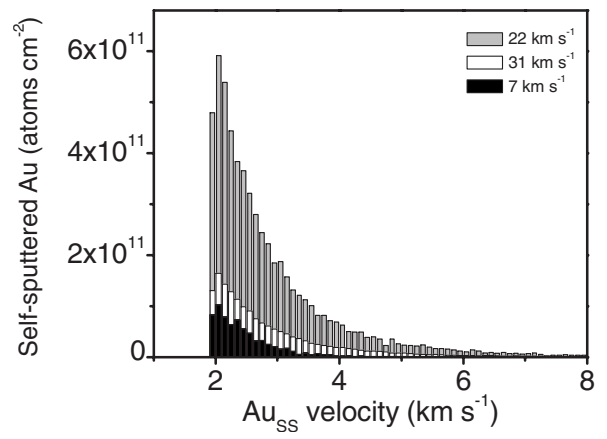

FIG. 9. Calculated elemental velocity distributions of atoms self-sputtered by incident $\mathrm{Au}^{+}$ions having velocities of $7 \mathrm{~km} \mathrm{~s}^{-1}$, $22 \mathrm{~km} \mathrm{~s}^{-1}$, and $31 \mathrm{~km} \mathrm{~s}^{-1}$. Data correspond to a laser fluence of $9.0 \mathrm{~J} \mathrm{~cm}^{-2}$. 
elemental velocity distributions for SS neutrals generated by $\mathrm{Au}^{+}$ions reaching the substrate at three representative velocities $v: 7 \mathrm{~km} \mathrm{~s}^{-1}$ (low), $22 \mathrm{~km} \mathrm{~s}^{-1}$ (medium), and $31 \mathrm{~km} \mathrm{~s}^{-1}$ (high). The values of $Q$ and $N(v)$ correspond to those obtained for $9.0 \mathrm{~J} \mathrm{~cm}^{-2}$ and are presented in Table I and Fig. 4, respectively. The highest density of self-sputtered neutrals is obtained for incident ions having an intermediate velocity of $v=22 \mathrm{~km} \mathrm{~s}^{-1}$. Finally, the velocity distribution of $\mathrm{SS}$ atoms or BS ions can be then evaluated as

$$
S_{i}\left(v_{z}^{\prime}\right)=\int_{v} s_{i}\left(v, v_{z}^{\prime}\right) d v=Q \int_{v} N(v) y_{i}\left(v, v_{z}^{\prime}\right) d v .
$$

*j.gonzalo@io.cfmac.csic.es

${ }^{1}$ Pulsed Laser Deposition of Thin Films, edited by D. B. Chrisey and G. K. Hubler (Wiley, New York, 1994).

${ }^{2}$ C. N. Afonso, J. Gonzalo, R. Serna, and J. Solis, in Laser Ablation and Its Applications, edited by C. R. Phipps (Springer, New York, 2007), Chap. 13.

${ }^{3}$ J. C. S. Kools, in Pulsed Laser Deposition of Thin Films, edited by D. B. Chrisey and G. K. Hubler (Wiley, New York, 1994), Chap. 19.

${ }^{4}$ R. Gupta, M. Weisheit, H.-U. Krebs, P. Schaaf, Phys. Rev. B 67, 075402 (2003).

${ }^{5}$ M. S. Al-Busaidy, O. E. Kusmartseva, and M. D. Crapper, Appl. Phys. A: Mater. Sci. Process. 79, 1453 (2004).

${ }^{6}$ J. Gonzalo, A. Perea, D. Babonneau, C. N. Afonso, N. Beer, J.-P. Barnes, A. K. Petford-Long, D. E. Hole, and P. D. Townsend, Phys. Rev. B 71, 125420 (2005).

${ }^{7}$ K. Seal, M. A. Nelson, Z. C. Ying, D. A. Genov, A. K. Sarychev, and V. M. Shalaev, Phys. Rev. B 67, 035318 (2003).

${ }^{8}$ R. del Coso, J. Requejo-Isidro, J. Solis, J. Gonzalo, and C. N. Afonso, J. Appl. Phys. 95, 2755 (2004).

${ }^{9}$ J. Q. Xiao, J. S. Jiang, and C. L. Chien, Phys. Rev. Lett. 68, 3749 (1992).

${ }^{10}$ S. J. Henley, J. D. Carey, and S. R. P. Silva, Appl. Phys. Lett. 88, 081904 (2006).

${ }^{11}$ A. Zenkevitch, J. Chevallier, and I. Khabelashvili, Thin Solid Films 311, 119 (1997).

${ }^{12}$ J. Lunney, Appl. Surf. Sci. 86, 79 (1995).
${ }^{13}$ B. Thestrup, B. Toftmann, J. Schou, B. Doggett, and J. G. Lunney, Appl. Surf. Sci. 197-198, 175 (2002).

${ }^{14}$ L. S. Dorneles, D. O’Mahony, C. B. Fitzgerald, F. McGee, M. Venkatesan, I. Stanca, J. G. Lunney, and J. M. D. Coey, Appl. Surf. Sci. 248, 406 (2005).

${ }^{15}$ E. Irissou, B. Le Drogoff, M. Chaker, and D. Guay, J. Appl. Phys. 94, 4796 (2003).

${ }^{16}$ E. van de Riet, J. C. S. Kools, and J. Dieleman, J. Appl. Phys. 73, 8290 (1993).

${ }^{17}$ S. Fahler, K. Sturm, and H.-U. Krebs, Appl. Phys. Lett. 75, 3766 (1999).

${ }^{18}$ K. Sturm and H.-U. Krebs, J. Appl. Phys. 90, 1061 (2001).

${ }^{19}$ J. G. Hidalgo, R. Serna, E. Haro-Poniatowsky, and C. N. Afonso, Appl. Phys. A: Mater. Sci. Process. 79, 915 (2004).

${ }^{20}$ Y. Nakata, W. K. Kummuduni, T. Okada, and M. Maeda, Appl. Phys. Lett. 64, 2599 (1994).

${ }^{21}$ T. Okada, Y. Nakayama, W. K. Kummuduni, and M. Maeda, Appl. Phys. Lett. 61, 2368 (1992).

${ }^{22}$ D. B. Geohegan, Appl. Phys. Lett. 62, 1463 (1993).

${ }^{23}$ J. Siegel, G. Epurescu, A. Perea, F. J. Gordillo-Vázquez, J. Gonzalo, and C. N. Afonso, Opt. Lett. 29, 2228 (2004).

${ }^{24}$ J. C. Ehrhardt and S. P. Davis, J. Opt. Soc. Am. 61, 1342 (1971).

${ }^{25}$ J. F. Ziegler, Nucl. Instrum. Methods Phys. Res. B 219, 1027 (2004).

${ }^{26}$ K. L. Saenger, Process. Adv. Mater. 3, 63 (1993).

${ }^{27}$ O. L. Landen, R. J. Winfield, D. D. Burgess, J. D. Kilkenny, and R. W. Lee, Phys. Rev. A 32, 2963 (1985). 